\title{
DSDMAC: Dual Sensing Directional MAC Protocol for Ad Hoc Networks with Directional Antennas
}

\author{
Ahmad Ali Abdullah, Member, IEEE, Lin Cai, Senior Member, IEEE, and Fayez Gebali, Senior Member, IEEE
}

\begin{abstract}
Applying directional antennas in wireless ad hoc networks can theoretically achieve higher spatial multiplexing gain and, thus, higher network throughput. However, in practice, deafness, hidden-terminal, and exposed terminal problems are exaggerated with directional antennas, and they cause the degradation of the overall network performance. Although there are several random-access-based medium-access control (MAC) protocols being proposed in the literature for networks with directional antennas, the deafness, hidden-terminal, and exposed terminal problems have yet to be fully solved. In this paper, we present a new MAC protocol called the dual-sensing directional MAC (DSDMAC) protocol for wireless ad hoc networks with directional antennas. Different from existing protocols, the DSDMAC protocol relies on the dual-sensing strategy to identify deafness, resolve the hidden-terminal problem, and avoid unnecessary blocking. The integrity of the DSDMAC protocol is verified and validated using $\mathrm{Spin}$, which is a formal protocol verification and validation tool. We further develop an analytical framework to quantify the performance of the DSDMAC protocol and conduct extensive simulations, which verify the accuracy of the analysis. The protocol verification, analysis, and simulation results show the robustness and superior performance of the DSDMAC protocol, which can achieve a much higher network throughput and lower delay utilizing the spatial multiplexing gain of the directional antennas. The results presented in this paper show that the proposed DSDMAC protocol can substantially outperform the state-of-the-art protocols.
\end{abstract}

Index Terms-Access protocols, ad hoc networks, computer simulation, directional antennas, multiple access, system analysis and design.

\section{INTRODUCTION}

$\mathbf{U}$ SING directional antennas, a higher antenna gain can be achieved, which results in a higher data rate, a larger transmission range, and/or a less transmission power. There are many applications using directional antennas. Vehicular networks, for example, are a natural application since the vehicular traffic usually follows a straight line. Millimeter-wave communications also use directional antenna to combat severe path loss [2]. When used in a network, directional antennas can reduce the number of blocked nodes and achieve higher spatial reuse. However, effective medium-access control (MAC) protocols

Manuscript received February 28, 2011; accepted January 26, 2012. Date of publication February 7, 2012; date of current version March 21, 2012. This paper was presented in part at the 2010 IEEE 72nd Vehicular Technology Conference, Ottawa, ON, Canada, September 6-9. The review of this paper was coordinated by Prof. P. Langendoerfer.

A. A. Abdullah is with Systems Xcellence Inc., Victoria, BC V8X 4A3, Canada (e-mail: ahmad.ali.abdullah@gmail.com).

L. Cai and F. Gebali are with the Department of Electrical and Computer Engineering, University of Victoria, Victoria, BC V8W 3P6, Canada (e-mail: cai@ece.uvic.ca; fayez@ece.uvic.ca).

Digital Object Identifier 10.1109/TVT.2012.2187082 that support the directional antenna face several challenges. In particular, the hidden-terminal, exposed-terminal, and deafness problems severely affect network performance.

Different from the situation with omnidirectional antennas, hidden terminals in networks with directional antennas are located near the source node, as they may not hear the source's transmissions; therefore, they may initiate transmissions, which lead to collisions. Deafness, on the other hand, occurs when a targeted destination does not reply when it is transmitting or receiving at a different direction. If it is not handled, failed transmissions due to deafness might be treated as collisions by the source node. Even worse, the source node may conclude that the destination node is unreachable.

Using directional antennas in ad hoc networks poses challenging problems for MAC protocol design. The main contributions of this paper are of threefold: First, we propose a dual-sensing directional MAC protocol (DSDMAC) for networks with directional antennas. The protocol helps to improve the throughput and delay performance of the wireless networks by minimizing the negative effect of the hidden-terminal, exposed-terminal, and deafness problems. The protocol uses a noninterfering out-of-band busy-tone signal combined with sensing the activity on the actual data channel to identify deafness situations and to avoid unnecessary blocking. In addition, the protocol avoids the asymmetry-in-gain problem introduced by other solutions. Second, the integrity of the DSDMAC protocol is verified using Spin, which is a formal protocol verification tool. Finally, a framework for throughput and delay analysis of wireless ad hoc networks using directional antennas is presented. The accuracy of the analysis is validated by simulation results, showing the advantages of applying the DSDMAC protocol, which can outperform the state-of-the-art protocols by $15 \%-184 \%$.

The rest of this paper is organized as follows: Section II reviews the related work. In Section III, we define the MAC design issues using directional antennas and the system model. The DSDMAC is introduced in Section IV, followed by the protocol validation in Section V. The performance analysis and simulation results are presented in Sections VI and VII, respectively. Concluding remarks are given in Section VIII.

\section{RELATED WORK}

Random-access-based MAC protocol design and analysis for ad hoc networks has attracted extensive research [3]-[6]. We focus on those considering directional antenna, which can be classified into the nonbusy-tone-based protocols and the busytone-based protocols, as discussed here. 


\section{A. Nonbusy-Tone-Based Protocols}

The directional MAC (DMAC) proposed in [7] is one of the earliest protocols that support directional antenna. Based on a modified 802.11 MAC protocol, DMAC uses a persector blocking mechanism to block a sector once it senses a request-to-send (RTS) or clear-to-send (CTS) packet. A node can transmit its RTS packet in an omnidirectional fashion when none of its sectors is blocked; otherwise, it beams toward its destination. The omnidirectional transmissions may cause unnecessary blocking, and the protocol requires knowledge of neighbors' locations.

In [8], it is suggested that RTS/CTS packets be exchanged in an omnidirectional fashion (ORTS/OCTS) using all available sectors. After a successful ORTS/OCTS handshake, the data and Acknowledgment (ACK) are transmitted in the directions from which the OCTS/ORTS are received at the maximum power. The protocol is efficient in minimizing the hiddenterminal problem. However, it creates a severer exposedterminal problem and cannot handle the deafness problem.

In [9], a multihop RTS MAC (MMAC) protocol is proposed where all packets including RTS/CTS should use directional transmission (DRTS/DCTS). Nodes, however, may listen in an omnidirectional mode while they are idle. The deafness problem still exists as not all neighboring nodes can receive the DRTS and DCTS.

The Directional Virtual Carrier Sensing protocol was proposed by in [10], which assumed a steerable antenna system to point at any specified direction. Each node maintains a list of neighbors and their directions based on the address of arrival (AoA) of any sensed signal. The AoA information is used to directly beam RTS packets to their destinations. If no location information exists, the RTS packets are omnidirectionally transmitted. A directional version of the network allocation vector is maintained for channel reservation. The protocol handles some basic functions required to support the directional antenna, and it cannot handle the hidden-terminal and deafness problems.

The protocols in [11] and [12] suggested a circular directional RTS in which an RTS packet has to be transmitted multiple times in each direction. This helps to identify the location of the source node by its intended destination who replies a CTS packet at the direction of the source. Sending the RTS packet at all possible directions helps to notify the neighbors about the intended communication. However, this would not eliminate the deafness problem. The protocols also require synchronization mechanisms and cause undesired waste of time. In addition, the previous RTS/CTS-based mechanisms cannot be used for multicasting and broadcasting [13].

\section{B. Busy-Tone-Based Protocols}

Using a busy tone to enhance the MAC protocol has been an active topic [13]-[18]. The tone-based DMAC (ToneDMAC) protocol proposed in [14] uses two separated channels, i.e., a data channel and a control channel. While the data channel is used to transmit the RTS/CTS/DATA/ACK packets, the control channel is used to transmit a busy-tone signal. A unique busy tone is assigned to each wireless node, and therefore, it can be identified, and each node should maintain a hash function for all neighbors' locations. When a source node has data to transmit, it transmits a directional RTS packet toward its destination immediately after sensing the medium at the intended direction. The destination node in response replies with a directional CTS packet back to the source node. The source and destination nodes continue with exchanging the actual data at the specified directions, and meanwhile, they omnidirectionally transmit a busy tone. If the source node detects a busy tone rather than receive a CTS packet, it then concludes a deafness situation. The protocol can identify some deafness situations; however, there are chances to miss the busy-tone signal from either or both the source and destination nodes, which do not guarantee a deafness-free protocol. In addition, to avoid the hidden-terminal problem, the busy-tone signal needs to be simultaneously transmitted as the RTS packet, and it also needs to be sensed before any other transmission.

In [15], the busy-tone signal to be transmitted by the destination node toward the direction of the source node only is proposed. The communication first starts with a DRTS/DCTS packet exchange in a directional manner. The redundant busytone signal would serve as another way to inform other nodes of the ongoing transmission in case they missed the DCTS packet. However, the deafness problem, which degrades the performance of the protocol, has not been addressed.

Dual-Busy-Tone Multiple Access with Directional Antennas (DBTMA/DA), which was proposed by Huang et al. [16], is a modified version of the Dual Busy-Tone Multiple Access (DBTMA) in [17] to accommodate the nodes with directional antennas. As in the original DBTMA, the DBTMA/DA uses two distinctive busy tones: a transmitter's busy tone $\left(B T_{t}\right)$ and a receiver's busy tone $\left(B T_{r}\right)$. The receiver turns on its $B T_{r}$ upon receiving the RTS packet, whereas the transmitter turns on its $B T_{t}$ upon receiving the CTS packet. Therefore, hidden terminals are notified after the CTS is being transmitted by the receiving node, leading to a large gap during which several collisions may occur.

In summary, how to solve the deafness problem and minimize the hidden terminal and exposed terminal problems for MAC protocol design is still an open issue, which motivates us to propose the DSDMAC protocol.

\section{Problem Definition and System Model}

\section{A. Hidden- and Exposed-Terminal Problem}

Unlike the omnidirectional counterpart, in directional antenna networks, the hidden terminals are located close to the source node. Theoretically, all nodes that are located within the destination node's coverage area and are away from the source node's coverage area are hidden terminals. The shaded area $A_{h}$ in Fig. 1(a) indicates the area at which hidden terminals may exist. Hidden terminals can severely degrade the network performance. Unfortunately, the standard RTS/CTS mechanism fails to completely solve the problem, as nodes in $A_{h}$ may initiate transmissions during the time the source node transmits the RTS, as discussed in [1] and [19].

The exposed-terminal problem needs more attention in directional antenna networks. For example, if using ORTS and/or 


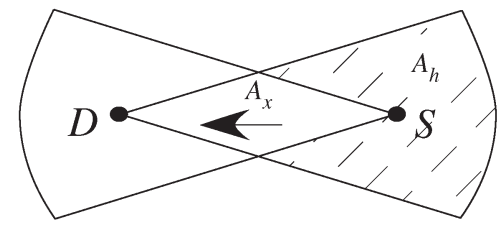

(a)



(b)

Fig. 1. Directional antenna problems. (a) Hidden terminals. (b) Deafness.

OCTS, nodes will unnecessarily block the sectors that can be used for concurrent transmissions; thus, it will waste the chance for higher spatial multiplexing gain, which defeats the purpose of using directional antenna.

\section{B. Deafness Problem}

The deafness problem occurs when nodes use directional antennas in ad hoc networks. It happens when a source node fails to communicate with its intended destination, which is pointing at a different direction for transmitting or receiving. For example, node $E$ in Fig. 1(b) is trying to communicate with node $S$ whereas $S$ is beamformed toward node $D$. As a result, node $E$ will double its backoff time for retransmission, as it concludes that a collision has occurred. Even worse, when node $E$ reaches the retry limit, it concludes that node $S$ is unreachable.

\section{Asymmetry-in-Gain Problem}

When using two different types of transmissions using the same antenna, e.g., a directional transmission for data packets and an omnidirectional transmission for control packets such as RTS/CTS, different transmission ranges lead to the asymmetryin-gain problem. The transmission range of a directed signal and the transmission range of an omnidirectional radiated signal are not identical. As a result, the control packets omnidirectionally transmitted will not reach all the desired nodes.

\section{Antenna Model}

A directional antenna is thought of to be an isotropic antenna with a constant gain over a certain angle $\Omega_{A}$, as shown in Fig. 2(a). Since we are interested in using the antenna in the azimuth plane, the radiation pattern presented in Fig. 2(b) shows the main pattern attributes. The pattern consists of the main lobe at the direction of the maximum radiation intensity and other minor sidelobes. The half-power beamwidth (or simply beamwidth) is the angle between the edges in the main lobe that are down from the maximum gain by $3 \mathrm{~dB}$.

In our antenna model, we assume an ideal antenna that has no sidelobes. According to Stutzman and Thiele [20], the area size of the sidelobes is much smaller than the area size of the main lobes. Therefore, the probability of finding wireless

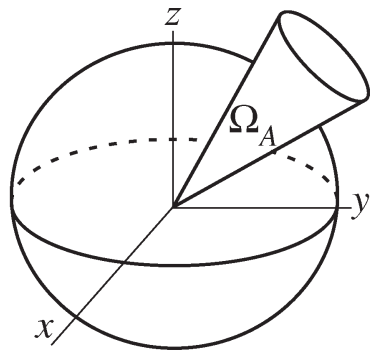

(a)

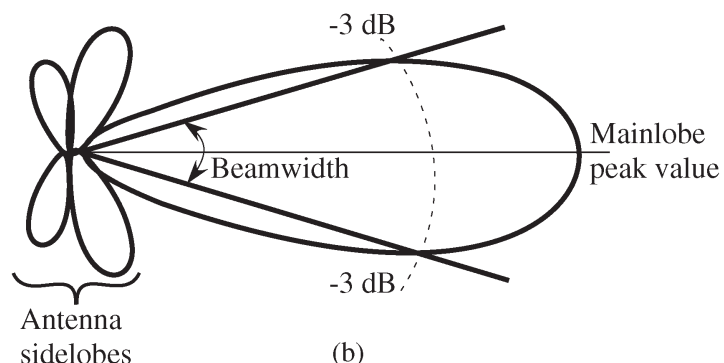

sidelobes

(b)

Fig. 2. Antenna pattern. (a) Beam solid angle $\Omega_{A}$. (b) Antenna power pattern.

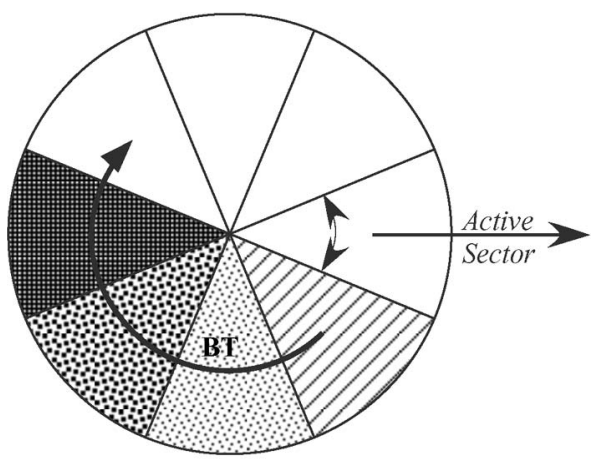

(a)

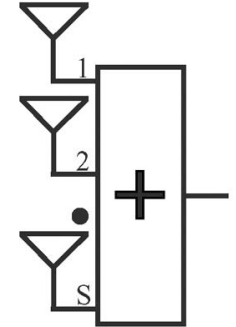

(b)

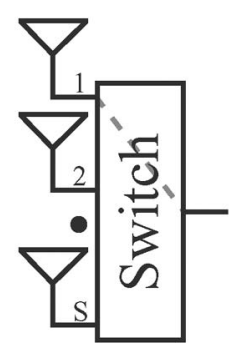

(c)
Fig. 3. Directional antenna model. (a) Antenna sectors. (b) Omnidirectional function. (c) Selecting a specific sector.

nodes within the sidelobes of the antenna is very small and can be ignored. The antenna has a constant gain within the beamwidth and zero outside. To cover all directions, we use $S \times \Theta \geq 2 \pi$, where $S$ is the number of antenna sectors and $\Theta$ is the beamwidth of a single antenna sector, as shown in Fig. 3(a). Sectors can be simultaneously used to provide an omnidirectional receiving function, as shown in Fig. 3(b), or they can be individually switched for a specific direction, as shown in Fig. 3(c). An antenna controller is assumed. The controller keeps track of the directions from which a maximum signal power is received. It then informs the higher layers about the sector of the received signal. The switching within the antenna controller can be achieved by using very fast analog 


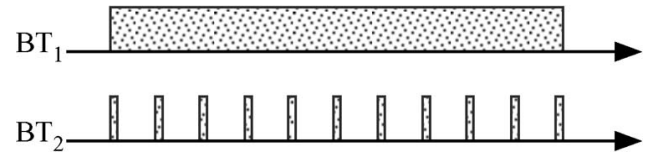

Fig. 4. Busy-tone signal patterns.

CMOS multiplexers/demultiplexers, which have a transition time of less than 217 ns (e.g., ADG5408/ADG5409, four-/ eight-channel multiplexers). This transition time is less than the signal propagation delay. Therefore, the short interframe space (SIFS) defined in the 802.11 standard is long enough for the antenna being switched between transmitting and receiving modes. Data packets are transmitted using one sector, and the busy-tone signal may be rotationally transmitted one sector at a time over all available (nonblocked) sectors.

\section{E. Busy-Tone Signal}

The busy-tone signal is a noninterfering sine-wave signal used to let other nodes be aware of an ongoing transmission. In this paper, two different patterns are used: a continuous pattern, which is referred to as $B T_{1}$, and an ON/OFF pattern, which is referred to as $B T_{2}$. The two patterns are shown in Fig. 4. $B T_{1}$ and $B T_{2}$ are used for different purposes, which will be discussed in more detail in the next section. When more than one busy-tone signals are constructively interfering with each other, a fall-back to a $B T_{1}$ results.

\section{F. Effect of Mobility on MAC Protocols}

Mobility in the wireless multihop ad hoc networks may affect both routing and MAC protocols. While the routing protocols need to deal with the change in connectivity among the wireless nodes, MAC protocols can only be affected if the time scale of the MAC frame transmission is similar to the time scale of the changes in the network. However, from the following example, the time to complete a MAC transaction is very short compared with the time scale of network connectivity changes due to mobility. Considering a vehicle traveling at a speed of $90 \mathrm{~km} / \mathrm{h}$ in a highway, the time required to move the vehicle by $1 \mathrm{~m}$ is $40 \mathrm{~ms}$, whereas a packet of a 12000 -bit payload requires only 2.2-ms transmission time by an IEEE 802.11 link with a data rate of $11 \mathrm{Mb} / \mathrm{s}$. Accordingly, the mobility has a very limited impact on the MAC protocols, and it has a greater impact on the routing protocols, which are beyond the scope of this paper.

\section{Dual-Sensing Directional Medium-Access CONTROL PROTOCOL}

The proposed DSDMAC protocol uses two well-separated wireless channels, i.e., a data channel and a busy-tone channel. The data channel carries the data packets and the RTS, CTS, and ACK packets on a specified direction (DRTS, DCTS, DDATA, and DACK). On the other hand, the busy-tone channel will be used to transmit a sine-wave busy-tone signal on all other directions. Only the source and destination nodes will transmit the busy-tone signal. The protocol assumes that the directions of all reachable destinations or forwarders are predetermined (during the node discovery period for example).

\section{A. Transmitting and Receiving With DRTS/DCTS}

When the link layer of a wireless node receives data packets from its higher layer, it senses the activity on the data channel at the specified direction. If the specified sector is not blocked, the data channel is idle, and no $B T_{1}$ is present, it immediately transmits a DRTS packet and turns on its $B T_{1}$ signal at all other directions. In case a $B T_{1}$ was sensed, other nodes should postpone any DRTS until $B T_{1}$ disappears. Otherwise, the source node waits until the tagged sector is unblocked and becomes idle for the period of a distributed interframe space (DIFS). It then generates a random backoff interval before transmitting its DRTS packet. The backoff interval is randomly chosen between 0 and $C W-1$, where $C W$ is the initial contention window size. The backoff counter is always frozen whenever the node senses an activity on the data channels at the specified direction or whenever the sector at the specified direction is blocked (e.g., by DRTS/DCTS from other nodes). Once the backoff counter reaches zero, the node transmits its DRTS packet at the specified direction and turns on its $B T_{1}$ signal over all other directions. The source node should change the $B T_{1}$ to $B T_{2}$ after finishing the DRTS packet transmission plus an SIFS duration. We will discuss the reason for the BT switching shortly.

On the destination side, the receiving node (to which the DRTS packet is addressed) replies after an SIFS period with a DCTS packet at the specified direction and turns on its $B T_{2}$ signal at all other directions. It then waits for the data packet. Once the data packet is successfully received, the destination node acknowledges it by sending a DACK packet at the same direction. After that, it turns off its busy-tone signal.

$B T_{1}$ is used to avoid the hidden-terminal problem. Because DRTS cannot be sensed by the nodes in the hidden terminal area (see $A_{h}$ in Fig. 1), these nodes can avoid initiating a new DRTS when they sense the $B T_{1} . B T_{1}$ can be turned off after the DRTS plus an SIFS because the nodes in $A_{h}$ can sense the CTS to avoid collision.

$B T_{2}$ is used to solve the deafness problem. When a node is directionally transmitting or receiving, it will not be able to respond to other DRTS. When a source notices a failed DRTS, it should check whether there is a $B T_{2}$ from the receiver's direction. If not, it concludes that there is a collision for the DRTS; otherwise, the receiver is busy in other transmissions. Therefore, if the source node does not receive a DCTS packet within a specified CTS-Timeout interval and it senses a $B T_{2}$, it reschedules the transmission of the packet for a later time (after the busy tone has disappeared) without doubling its backoff $\mathrm{CW}$; if there is no $B T_{2}$, it reschedules the transmission of the packet for a later time and doubles its backoff $\mathrm{CW}$.

Once the source node successfully receives the DCTS, it directionally transmits the data packet. After that, if the source node does not receive a DACK packet within a specified ACK-timeout interval or it detects a transmission of a different packet, it reschedules the transmission of the data packet for a later time and doubles its backoff $\mathrm{CW}$.

\section{B. DNAV Mechanism}

When a node receives a valid DRTS packet, it should set its per sector directional network allocation vector (DNAV) 


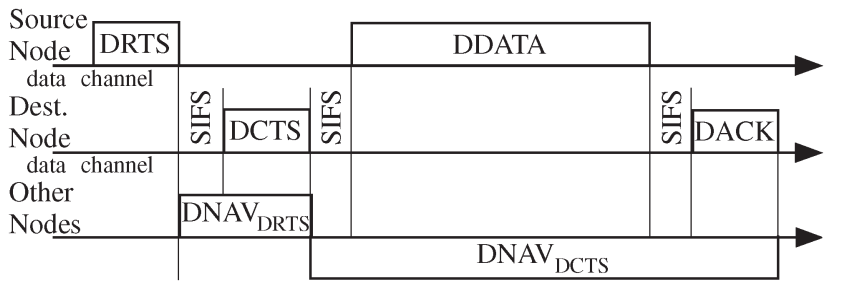

Fig. 5. DNAV setting.

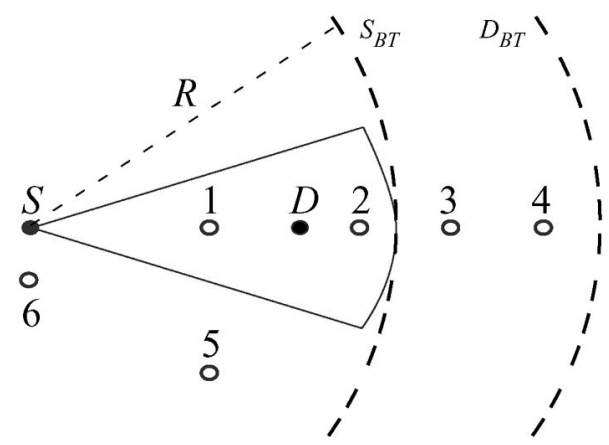

Fig. 6. Case study.

timers. It also should block all of its sectors for a period with a duration of SIFS $+D C T S$, as shown in Fig. 5. We call this time $D N A V_{\text {DRTS }}$ time. Unless a DCTS packet is received, the node should unblock its antenna sectors when the $D N A V_{\text {DRTS }}$ timer is expired. If a DCTS packet is received, then only the receiving sector and the sector from which a previously DRTS packet is received (if applicable) should remain blocked for a period with a duration of $2 \times S I F S+D D A T A+D A C K$, so the node will not initiate any transmissions to interfere the ongoing transmission. We call this time $D N A V_{\mathrm{DCTS}}$ time, as shown in Fig. 5. Using this DNAV design, we can minimize the exposed-terminal problem without increasing the collision probability.

\section{Case Study and State Transitions of DSDMAC}

To further illustrate how the DSDMAC meets its design goal, we use an example with the network configuration shown in Fig. 6. The source and destination nodes $(S$ and $D)$ are marked with solid dots. Nodes 1, 2, 3, and 4 are located on the same line connecting node $S$ and node $D$. Nodes 5 and 6 are located at different directions. The dashed curve $S_{\mathrm{BT}}$ marks the circular region of node $S$ 's busy-tone signal range with a radius of $R$. Likewise, the dashed curve $D_{\mathrm{BT}}$ marks the circular range of node $D$ 's busy-tone signal. The message exchanges among these nodes are shown in Fig. 7, where the arrow within each packet indicates the direction used to transmit that packet.

As shown in Fig. 7, node $S$ waits until nodes 1 and 2 finish their communication. Meanwhile, nodes 3 and 4 may independently start their communication because the direction from node 3 to node 4 is not blocked. When node $S$ senses no $B T_{1}$ in the busy-tone channel and no new activities in the data channel, and its corresponding antenna sector toward $D$ is not blocked, it starts its transmission to node $D$ after a backoff period. Any further transmission from node 1 to node 2 must be deferred until node $S$ finishes. However, node 5 can independently start its transmission toward node 2 because the direction from node 5 to node 2 is not blocked. As a hiddenterminal, node 6 will be blocked from transmission while it hears the $B T_{1}$ from $S$, and it will then receive D's DCTS and avoid collisions.

The state transition diagram for the DSDMAC protocol is shown in Fig. 8. Although the states are self-explanatory, the following highlights the most important states, which are different from the IEEE 802.11 MAC protocol. The system is initially in its idle state until a packet arrives from the higher layers or a packet arrives from another node. When a packet arrives from the higher layers, there is no new activity in the data channel, no $B T_{1}$ is sensed, and the corresponding antenna sector is not blocked, the system moves to the "Send DRTS \& start BT" state and sets a timer to wait for the DCTS. Otherwise, the system moves to the "Wait" state until the channels become idle and the sector is unblocked; then, it moves to the "Backoff" state.

In case the timer expired without receiving a DCTS packet and a $B T_{2}$ signal is presented, the system skips the "Double backoff counter" state, as it concludes that its destination might be busy toward other directions. In this case, the system will directly go to the "Wait" state. This transition helps the DSDMAC protocol to avoid the deafness problem.

\section{PRotocol Validation}

The validation of the DSDMAC protocol is carried out using a popular open-source tool called Simple Promela INterpreter (Spin), which is capable of analyzing PROtocol/PROcess MEta LAnguage (Promela) code to detect design errors, such as deadlocks and other violations.

To examine whether the DSDMAC protocol meets the suggested requirements such as deafness free, a traversal exploration through the state space generated by the DSDMAC protocol (see Fig. 8) is required. When the applied model is complex, the state explosion problem will be encountered. Therefore, we have applied validation techniques such as partial state exploration to reduce the complexity [21].

Promela is specifically designed to model network protocols and multithreaded programs. For the purpose of validating the DSDMAC protocol, we have modeled our network using a limited number of wireless nodes to reduce the network state space. This should not affect the integrity of the validation process since the properties that we are willing to test mainly depend on the locations of the wireless nodes rather than the size of the network. Fig. 9 shows a sample network used for our validation process. The solid circles represent the wireless nodes, and the arrows represent the traffic flow direction.

As an example, in the following, we demonstrate how to use Spin to verify whether the existing and proposed MAC protocols are deafness free and blocking free. To verify the deafness-free property, given that the wireless nodes are precisely allocated at locations that guarantee no collisions or hidden-terminal situations, an RTS packet should always be 




Fig. 7. DSDMAC: DRTS/DCTS/DDATA/DACK and BT setting.

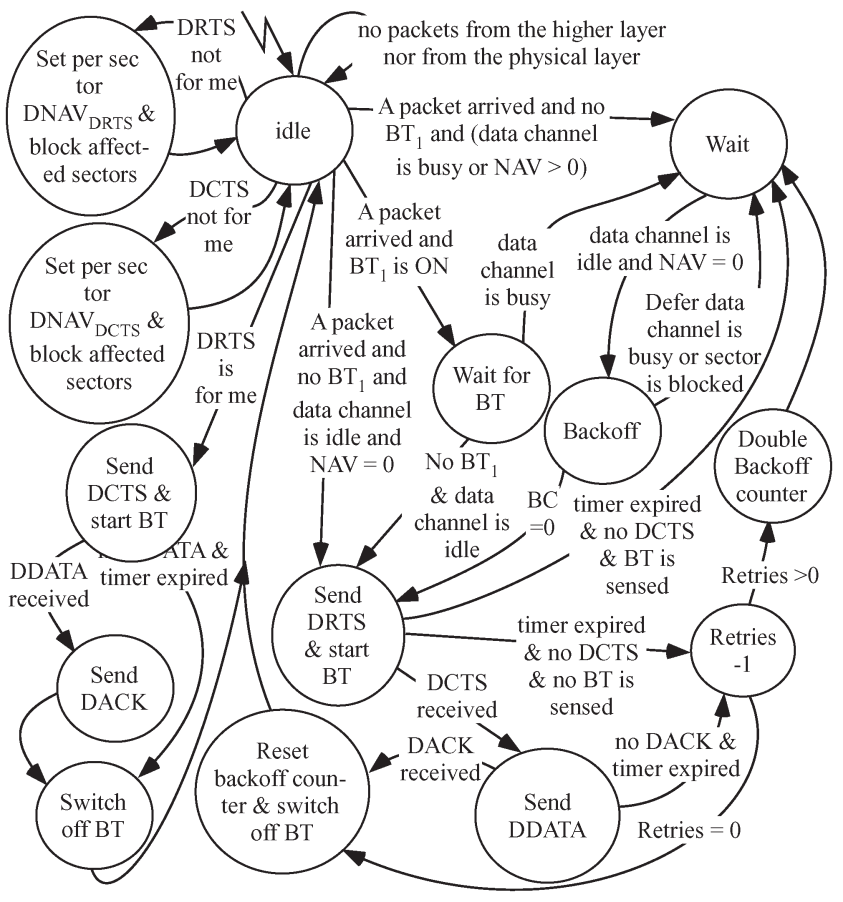

Fig. 8. DSDMAC system state transition diagram.



Fig. 9. Sample network used for validation.

followed by a CTS or by hearing a busy-tone signal. This can be written in a formal temporal claim as

$$
\square(\mathrm{RTS} \diamond(\mathrm{BT} \| \mathrm{CTS}))
$$

and read as follows: always RTS, then eventually BT or CTS. A similar formula can be introduced to capture the blocking
TABLE I

SPIN VERIFICATION RESULTS

\begin{tabular}{l|l|l}
\hline Protocol & Blocking free & Deafness free \\
\hline DMAC [7] & Not valid & Not valid \\
MMAC [9] & Not valid & Not valid \\
Tone DMAC [14] & Valid & Not valid \\
DBTMA/DA [16] & Not valid & Valid \\
DSDMAC & Valid & Valid \\
\hline
\end{tabular}

situations. Let $D_{x y}$ denote a directional transmission from node $x$ to node $y$, and let $B_{x y}$ denote a blocked direction from node $x$ to node $y$. A blocking claim can be expressed as

$$
\begin{aligned}
& \square\left(D_{12} \square\left(B_{34} \| B_{56}\right)\right) \\
& \square\left(D_{34} \square\left(B_{12} \| B_{56}\right)\right) \\
& \square\left(D_{56} \square\left(B_{12}\left\|B_{34}\right\| B_{32}\right)\right) .
\end{aligned}
$$

Spin results are summarized in Table I. The results show that omnidirectionally transmitting the RTS/CTS in the DMAC protocol can block node 5 from transmission when node 1 or node 3 starts a transmission to node 2. Deafness situations are also likely occurring if node 3 misses node 2's CTS. The MMAC has also failed the test. The omnidirectional reception of the RTS packets, as stated by the protocol, will block node 6 from receiving node 5's RTS when node 1 transmits toward node 2 . In addition, the deafness situation occurs when node 3 tries to send a packet to node 2 while node 2 is receiving from node 1 . The ToneDMAC, on the other hand, shows good results on handling the blocking problem. However, according to the discussion in Section II, deafness situations are not completely eliminated. The DBTMA/DA protocol is vulnerable to the blocking problem since a source node is prevented from transmission at the presence of the $B T_{r}$ signal. Deafness, however, was not reported. Finally, the validation results show that DSDMAC can handle both deafness and blocking as expected, and thus, it outperforms the other protocols. Next, we develop an analytical framework to quantify the protocol performance. 


\section{Performance Analysis}

In this section, we develop the analytical models to quantify the throughput and the delay in a wireless network using the directional antenna. In our analysis, we make the following assumptions: All wireless nodes are identical and equipped with the same type of antennas. Each node has $S$ antenna sectors, which can be individually switched toward a specific direction or can be summed together for an omnidirectional reception. It is also assumed that a busy-tone signal can be transmitted over all of the unused sectors during communications. Nodes are randomly distributed according to a Poisson distribution in a 2-D space, and all active nodes are saturated, i.e., their data buffers are always nonempty.

\section{A. Throughput Analysis}

Given the exponential backoff strategy used in the MAC protocol, we first apply Bianchi's equation for the packet transmission probability [3]

$$
a(p)=\left(1+\frac{1-p}{1-p^{m+1}} \sum_{i=0}^{m} p^{i} E\left[b_{i}\right]\right)^{-1}
$$

where $p$ is the packet collision probability, $m$ is the maximum number of backoff stages, and $E\left[b_{i}\right]=C W_{i} / 2$ is the average value of the backoff counter in stage $i$. See [3] for a detailed derivation of (1).

Different from Bianchi's work and its many follow-up works, the challenging part here is that we need to consider the more complicated situations associated with directional antenna. We assume that an active node chooses a sector (a direction) with probability $1 / S$. For a sensing slot, the tagged node chooses one sector at a time and is ready to transmit toward its destination with a probability of $a$, while other nodes are ready to transmit toward the same destination with a probability of $a / S$. Therefore, the probability that none of the other nodes is ready to transmit is given by

$$
P_{0}=\sum_{i=2}^{\infty}\left(1-\frac{a}{S}\right)^{i-1} \frac{\left(\lambda A_{S}\right)^{i}}{i !} e^{-\lambda A_{S}}
$$

where $\lambda$ is the node density in the network, and $A_{S} \approx\left(\pi R^{2} / S\right)$ is the sector area. Apart from the tagged node, the probability that one of the other wireless nodes is ready to transmit toward the destination node direction is given by

$$
P_{1}=\sum_{i=2}^{\infty}(i-1) \cdot \frac{a}{S} \cdot\left(1-\frac{a}{S}\right)^{i-2} \frac{\left(\lambda A_{S}\right)^{i}}{i !} e^{-\lambda A_{S}} .
$$

The wireless channel within the chosen sector is either idle or occupied by a successful or collided transmission. The channel is idle when none of the other nodes or the tagged node is ready to transmit or when there was less than two nodes in the area. Therefore, the idle probability is given by

$$
P_{\text {idle }}=(1-a) P_{0}+\left(1-p_{n}\right)-(1-a)\left(1-p_{n}\right) P_{0}
$$

where $p_{n}=1-\left(1+\lambda \pi R^{2}\right) e^{-\lambda \pi R^{2}}$ is the probability of finding two or more wireless nodes within the area. A successful transmission, however, occurs when either one of the other nodes or the tagged node are successfully transmitting, which is given by

$$
P_{s}=a P_{0} p_{n}+(1-a) P_{1} p_{n} .
$$

Finally, the collision probability is given by

$$
p=\left(1-P_{0}-P_{1}\right) p_{n}+a P_{1} p_{n} .
$$

We define the per-hop throughput as the total throughput within a circle area centered at the tagged node and with radius equal to its transmission range. Once the value of $a$ is computed using (1), the per-hop throughput can be computed as

$$
T h=\frac{P_{\mathrm{tr}} E[P]^{\prime}}{E[\text { Slot }]}
$$

where $E[P]^{\prime}=E[P](1+1 / C W$ min $)$ is the average amount of payload bits $P$ transmitted in one slot $\sigma$ [3], $P_{\mathrm{tr}}=a(1-$ p) $p_{n}$ is the success probability of the tagged node, and $E[$ Slot $]=P_{\text {idle }} \sigma+P_{s} T_{s}+p T_{c}$. The values $\sigma, T_{s}$, and $T_{c}$ are the periods of an empty slot, the time required to successfully transmit a data packet, and the wasted time due to a collision, respectively. They are protocol dependent. For instance, considering the IEEE 802.11 standard with RTS/CTS enabled, we have

$$
\begin{aligned}
T_{s}= & D R T S+S I F S+\delta+D C T S+S I F S+\delta+H \\
& +E[P]+S I F S+\delta+D A C K+D I F S+\delta \\
T_{c}= & D R T S+D I F S+\delta
\end{aligned}
$$

where $D R T S, D C T S$, and $D A C K$ are the transmission durations of directional RTS, CTS, and ACK packets, respectively; $\delta$ is the propagation delay; and $H$ and $P$ are the transmission durations of the packet header (PHY and MAC headers) and the packet payload, respectively.

\section{B. MAC Delay Analysis}

As in [19], we define the MAC delay as the time required for transmitting a data packet from the time it reaches the MAC sublayer (excluding queuing delay) to the moment it is received successfully. A packet transmission may involve up to $m$ trials (where $m$ is the maximum retry limit) until it can be successfully transmitted. Let $n_{a}$ be the average number of unsuccessful attempts. We have

$$
n_{a}=\frac{\sum_{n=0}^{m} n\left(1-P_{\mathrm{tr}}\right)^{n}}{\sum_{n=0}^{m}\left(1-P_{\mathrm{tr}}\right)^{n}} .
$$

We call each transmission attempt as a round, and each round can be divided into a number of slots. A slot can be either idle or busy for a successful/unsuccessful transmission. A failed round consists of a number of idle slots and a number of successful and unsuccessful slots by other nodes and ends with a failed slot by the tagged node. A successful round ends with a successful 
TABLE II

SYSTEM PARAMETERS

\begin{tabular}{ll|ll}
\hline Parameter & Value & Parameter & Value \\
\hline PHY & DSSS & Propagation delay $(\delta)$ & $1 \mu \mathrm{s}$ \\
$C W_{\text {min }}$ & 32 & $m$ & 7 \\
$C W_{\max }$ & 1024 & packet payload & 12000 bits \\
Channel data rate & $11 \mathrm{Mbps}$ & MAC header & $272 \mathrm{bits}$ \\
Basic data rate & $1 \mathrm{Mbps}$ & PHY header & $192 \mathrm{bits}$ \\
SIFS & $10 \mu \mathrm{s}$ & ACK & $304 \mathrm{bits}$ \\
DIFS & $50 \mu \mathrm{s}$ & RTS & $352 \mathrm{bits}$ \\
Slot Time $(\sigma)$ & $20 \mu \mathrm{s}$ & CTS & 304 bits \\
\hline
\end{tabular}

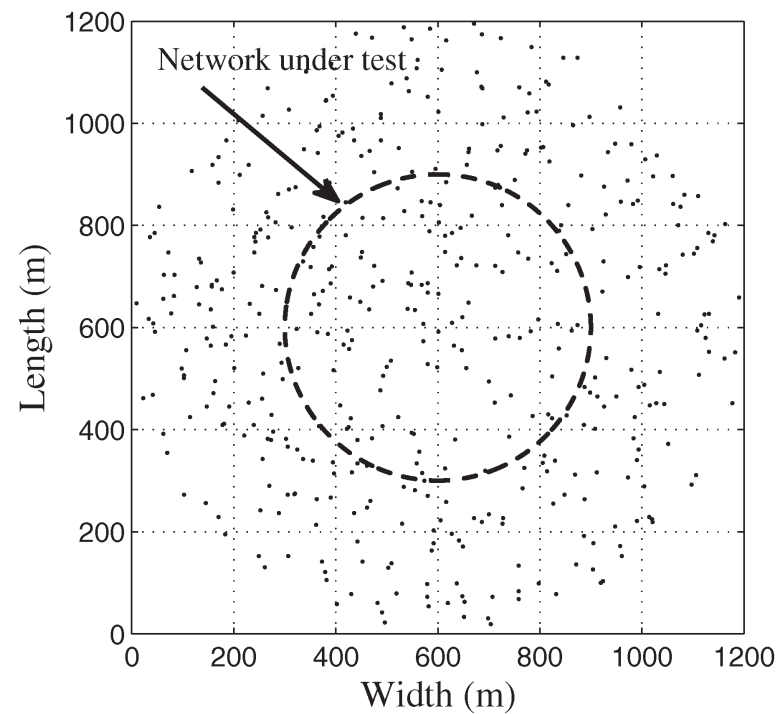

Fig. 10. Randomly picked snapshot from simulation runs showing node distribution in the wireless network.

transmission by the tagged node. Since each round ends with a transmission slot by the tagged node, the average number of slots per round is given by

$$
s=\sum_{i=0}^{\infty} i(1-a)^{i} a=\frac{1}{a}-1
$$

where $a$ is the probability that the tagged node is ready to transmit. Finally, the MAC delay is given by the average duration of a round $(s E[$ Slot $])$ multiplied by the average number of rounds $\left(n_{a}+1\right)$

$$
\text { delay }=\left(n_{a}+1\right) s E[\text { Slot }] .
$$

\section{Performance Evaluation}

In this section, we compare our analysis results with simulation results using a discrete event simulator. The system parameters used in both the simulations and the analysis are summarized in Table II. The wireless nodes are randomly distributed in a circular area with a radius of $300 \mathrm{~m}$ (which is twice of the transmission range) according to a 2-D Poisson distribution. All nodes are identical and use directional antennas in free space (no obstacles). To avoid the edge effect, we only considered the data collected from the nodes that are located in the center of the network, i.e., within $150 \mathrm{~m}$ from the center, as shown in Fig. 10. The wireless nodes are loaded with CBR traffic with rates high enough to achieve traffic saturation.



Fig. 11. Per-hop throughput, using one, four, eight, and 16 antenna sectors. (Line) Analytical results. (Error bar) 95\% confidence intervals of simulation results.

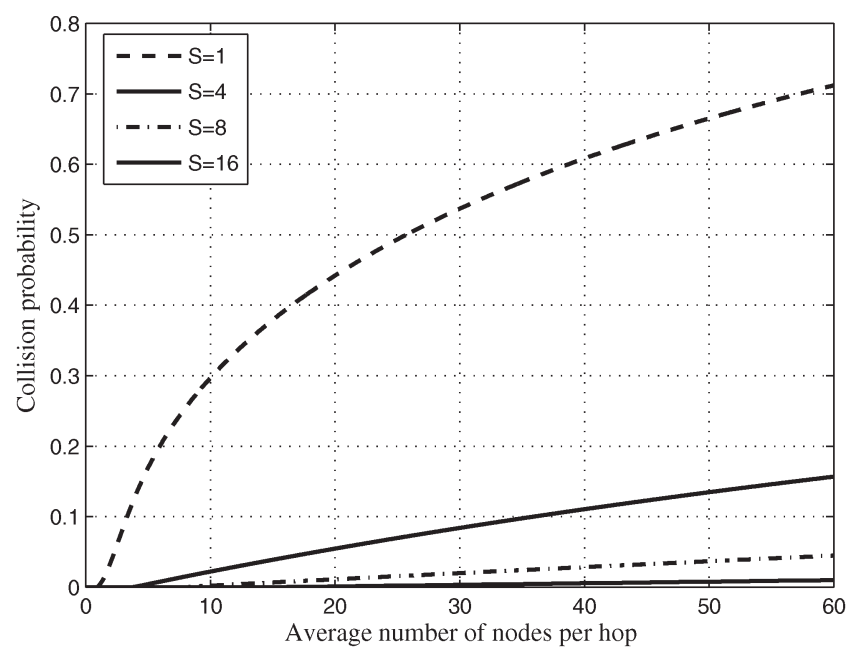

Fig. 12. Collision probabilities and analysis results.

In the following, we first compare the performance of the DSDMAC protocol for nodes with directional antennas with that of the standard IEEE 802.11 DCF protocol for nodes with omnidirectional antennas, in terms of per-hop throughput and MAC delay. Then, we compare the performance of DSDMAC and other DMAC protocols.

The per-hop throughputs with different numbers of antenna sectors are shown in Fig. 11. The lines are the analytical results, and the error bars represent the $95 \%$ confidence intervals of the simulation results. First, the accuracy of the analysis is validated by the simulation. Second, the results show that, with DSDMAC, more antenna sectors per node can result in a higher throughput. This is attributed to the reduction in the collision probabilities when more antenna sectors are used, as shown in Fig. 12.

In addition, given the DSDMAC protocol can appropriately deal with the deafness, hidden-terminal, and exposed terminal problems, much higher throughput can be achieved in a dense network due to the spatial multiplexing gain by using the directional antennas. As shown in Fig. 11, when the node density 
TABLE III

AgGregate CBR Multihop Throughrut

\begin{tabular}{|c|c|c|c|c|}
\hline DSDMAC & $\begin{array}{c}\text { ToneDMAC } \\
{[14]}\end{array}$ & $\begin{array}{c}\text { ZeroTone } \\
{[14]}\end{array}$ & $\begin{array}{c}\text { MMAC } \\
{[9]}\end{array}$ & $\begin{array}{c}\text { C-DMAC } \\
{[12]}\end{array}$ \\
\hline 5.4 & 4.7 & 4.1 & 3.2 & 1.9 \\
\hline
\end{tabular}

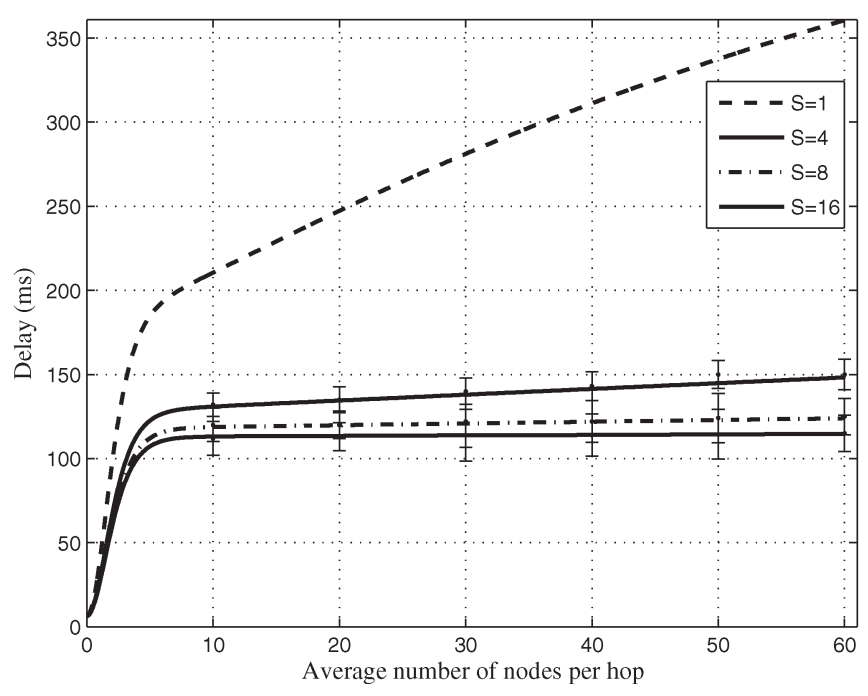

Fig. 13. MAC delay with one, four, eight, and 16 antenna sectors. (Line) Analysis results. (Error bar) $95 \%$ confidence intervals of simulation results.

is above 2 (nodes per hop), the throughput of " $s=1$ " (with IEEE 802.11 DCF) decreases fast, whereas the throughputs of " $s=4,8,16$ " (with DSDMAC) are more than twice higher at the density of 10 (nodes per hop), and the gaps are even larger when we further increase the density. Given the everincreasing demand of wireless services and the ever-dense wireless networks, it is desirable to adopt the proposed DSDMAC protocol to support directional transmission and reception in dense wireless networks.

The proposed DSDMAC is further compared to the state-ofthe-art DMAC protocols using the same network settings as in [14]. The results presented in Table III are the aggregated throughput of five flows averaged from 25 runs. The network consists of 30 nodes randomly placed in a region of $1500 \times$ $1500 \mathrm{~m}^{2}$ loaded with 512-B-packet-size CBR traffic. The source-destination node pairs are randomly chosen with the transmission range set to $300 \mathrm{~m}$ and the data rate set to $11 \mathrm{Mb} / \mathrm{s}$. The results in the table are presented in megabits per second and show that our proposed protocol can outperform, by $15 \%-184 \%$, the following DMAC protocols: ToneDMAC [14], ZeroTone [14], MMAC [9], and C-DMAC [12]. In addition, we believe that, when the node density is getting higher, our proposed protocol can have even higher performance gain, as our protocol is the only one not affected by the blocking and deafness problems according to the validation results in Table I.

With the proposed DSDMAC, the delay can also be improved by increasing the number of antenna sectors. Fig. 13 shows the delay versus the average number of nodes using one, four, eight, and 16 antenna sectors. The figure shows that, in addition to the higher throughput, a smaller MAC delay can be achieved using DSDMAC with directional antennas. With 60 nodes per hop, while the average delay ex- ceeds $350 \mathrm{~ms}$ for the wireless nodes equipped with omnidirectional antennas, the average delay remains below $150 \mathrm{~ms}$ when using four or more antenna sectors and applying the DSDMAC protocol.

In summary, if the MAC protocol is well designed to mitigate the deafness, hidden-terminal, and exposed-terminal problems, we can use directional antennas to achieve the spatial multiplexing gain and thus results in a higher network throughput and a lower delay.

\section{CONCLUSION}

In this paper, we have proposed a new MAC protocol called DSDMAC for wireless multihop ad hoc networks using directional antennas. The new protocol differs from the existing protocols by relying on the dual-sensing strategies to identify deafness, resolve the hidden-terminal problem, and avoid unnecessary blocking. The integrity of the DSDMAC has been verified using a formal protocol verification tool called Spin. Spin results have shown that the DSDMAC protocol outperforms the existing protocols. We have also presented the analytical model, which has been verified by simulation. The analytical and simulation results have shown that applying the DSDMAC protocol can greatly improve the performance of wireless networks using directional antennas.

\section{REFERENCES}

[1] A. Abdullah, L. Cai, and F. Gebali, "Enhanced busytone-assisted MAC protocol for wireless ad hoc networks," in Proc. IEEE VTC-Fall, 2010, pp. 1-10.

[2] L. X. Cai, L. Cai, X. Shen, and J. W. Mark, "Efficient resource management for mmWave WPANs," in Proc. IEEE WCNC, 2007, pp. 3816-3821.

[3] G. Bianchi, "Performance analysis of the IEEE 802.11 distributed coordination function," IEEE J. Select. Areas Commun., vol. 18, no. 3, pp. 535547, Mar. 2000.

[4] B. Kannhavong, H. Nakayama, A. Jamalipour, Y. Nemoto, and N. Kato, "A survey of routing attacks in MANET," IEEE Wireless Commun. Mag., vol. 14, no. 5, pp. 85-91, Oct. 2007.

[5] L. X. Cai, L. Cai, X. Shen, J. Mark, and Q. Zhang, "MAC protocol design and optimization for multihop ultra-wideband networks," IEEE Trans. Wireless Commun., vol. 8, no. 8, pp. 4056-4065, Aug. 2009.

[6] J. Chen, Q. Yu, P. Cheng, Y. Sun, Y. Fan, and X. Shen, "Game theoretical approach for channel allocation in wireless sensor and actuator networks," IEEE Trans. Autom. Control, vol. 56, no. 10, pp. 2332-2344, Oct. 2011.

[7] Y. B. Ko, V. Shankarkumar, and N. H. Vaidya, "Medium access control protocols using directional antennas in ad hoc networks," in Proc. IEEE INFOCOM, Mar. 2000, vol. 1, pp. 13-21.

[8] A. Nasipuri, S. Ye, J. You, and R. Hiromoto, "A MAC protocol for mobile ad hoc networks using directional antennas," in Proc. IEEE WCNC, Sep. 2000, vol. 3, pp. 1214-1219.

[9] R. R. Choudhury, X. Yang, N. H. Vaidya, and R. Ramanathan, "Using directional antennas for medium access control in ad hoc networks," in Proc. ACM MobiCom, 2002, pp. 59-70.

[10] M. Takai, J. Martin, A. Ren, and R. Bagrodia, "Directional virtual carrier sensing for directional antennas in mobile ad hoc networks," in Proc. ACM Mobihoc, 2002, pp. 183-193.

[11] E. Shihab, L. Cai, and J. Pan, "A distributed, asynchronous directionalto-directional MAC protocol for wireless ad hoc networks," IEEE Trans. Veh. Technol., vol. 58, no. 9, pp. 5124-5134, Nov. 2009.

[12] T. Korakis, G. Jakllari, and L. Tassiulas, "A MAC protocol for full exploitation of directional antennas in ad-hoc wireless networks," in Proc. ACM MobiHoc, 2003, pp. 98-107.

[13] Q. He, L. Cai, X. Shen, and P. H. Ho, "Improving TCP performance over wireless ad hoc networks with busy tone assisted scheme," EURASIP J. Wireless Commun. Netw., vol. 2006, no. 2, pp. 1-11, Apr. 2006. 
[14] R. R. Choudhury and N. H. Vaidya, "Deafness: A MAC problem in ad hoc networks when using directional antennas," in Proc. IEEE ICNP, 2004, pp. 283-292.

[15] S. S. Kulkarni and C. Rosenberg, "DBSMA: A MAC protocol for multihop ad-hoc networks with directional antennas," in Proc. IEEE PIMRC, Sep. 2005, vol. 2, pp. 1371-1377.

[16] Z. Huang, C. C. Shen, C. Srisathapornphat, and C. Jaikaeo, "A busy-tone based directional MAC protocol for ad hoc networks," in Proc. IEEE Milcom, 2002, pp. 1233-1238.

[17] J. Deng and Z. J. Haas, "Dual busy tone multiple access (DBTMA): A new medium access control for packet radio networks," in Proc. ICUPC, 1998, vol. 2, pp. 973-977.

[18] P. Wang, H. Jiang, and W. Zhuang, "A new MAC scheme supporting voice/data traffic in wireless ad hoc networks," IEEE Trans. Mobile Comput., vol. 7, no. 12, pp. 1491-1503, Dec. 2008.

[19] A. Abdullah, F. Gebali, and L. Cai, "Modeling the throughput and delay in wireless multihop ad-hoc networks," in Proc. IEEE GLOBECOM, 2009, pp. 1-6.

[20] W. Stutzman and G. Thiele, Antenna Theory and Design. Hoboken, NJ: Wiley, 1998.

[21] C.-T. Chou and D. Peled, "Formal verification of partial order reduction technique for model checking," J. Autom. Reasoning, vol. 23, no. 3, pp. 265-298, Nov. 1999.

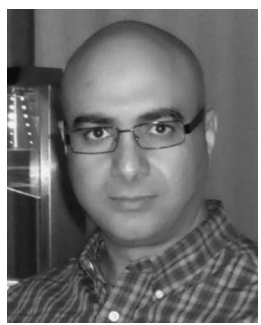

Ahmad Ali Abdullah (M'04) received the B.Sc. degree from the Higher Institute of Electronics, Beni-Walid, Libya, in 1991, the M.Eng. degree from Concordia University, Montreal, QC, Canada, and the Ph.D. degree from the University of Victoria, Victoria, BC, Canada, in 2011.

$\mathrm{He}$ is currently with Systems Xcellence Inc., Victoria. His research interests include wireless medium-access-control protocols.

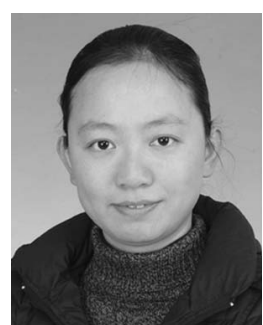

Lin Cai (S'00-M'06-SM'10) received the M.A.Sc. and $\mathrm{Ph} . \mathrm{D}$. degrees in electrical and computer engineering from the University of Waterloo, Waterloo, ON, Canada, in 2002 and 2005, respectively.

Since 2005, she has been an Assistant Professor and then an Associate Professor with the Department of Electrical and Computer Engineering, University of Victoria, Victoria, BC, Canada. She has been an Associate Editor for the EURASIP Journal on Wireless Communications and Networking, the International Journal of Sensor Networks, and the Journal of Communications and Networks. Her research interests include wireless communications and networking, with a focus on network protocol and architecture design supporting emerging multimedia traffic over wireless, mobile, ad hoc, and sensor networks.

Dr. Lin was a recipient of the Natural Sciences and Engineering Research Council of Canada NSERC Discovery Accelerator Supplement Grant in 2010, the Best Paper Award of the IEEE International Conference on Communications in 2008, and the Best Academic Paper Award of the IEEE Wireless Communications and Networking Conference in 2011. She has served as a Technical Program Committee Symposium Cochair for 2010 IEEE Globecom, and as an Associate Editor for the IEEE TRANSACTIONS ON Vehicular TeChNOLOGY and the IEEE TRANSACTIONS ON Wireless COMMUNICATIONS.



Fayez Gebali (S'76-M'80-SM'84) received the B.Sc. degree in electrical engineering (first-class honors) from Cairo University, Cairo, Egypt, the B.Sc. degree in mathematics (first-class honors) from Ain Shams University, Cairo, and the Ph.D. degree in electrical engineering from the University of British Columbia, Vancouver, BC, Canada, where he was a holder of the Natural Sciences and Engineering Research Council of Canada postgraduate scholarship.

$\mathrm{He}$ is currently a Professor of computer engineering and the Chair of the Department of Electrical and Computer Engineering, University of Victoria, Victoria, BC. His research interests include parallel algorithms, 3-D integrated-circuit design, hardware verification and security, and wireless communications. 